University of Texas Rio Grande Valley

ScholarWorks @ UTRGV

Psychological Science Faculty Publications and

Presentations

$5-2020$

\title{
Unique, Additive, and Interactive Effects of Types of Intimate Partner Cybervictimization on Depression in Hispanic Emerging Adults
}

Jorge I. Cantu

The University of Texas Rio Grande Valley

Ruby Charak

The University of Texas Rio Grande Valley

Follow this and additional works at: https://scholarworks.utrgv.edu/psy_fac

Part of the Psychology Commons

\section{Recommended Citation}

Cantu, J. I., \& Charak, R. (2020). Unique, Additive, and Interactive Effects of Types of Intimate Partner Cybervictimization on Depression in Hispanic Emerging Adults. Journal of Interpersonal Violence.

https://doi.org/10.1177/0886260520915552

This Article is brought to you for free and open access by the College of Liberal Arts at ScholarWorks @ UTRGV. It has been accepted for inclusion in Psychological Science Faculty Publications and Presentations by an authorized administrator of ScholarWorks @ UTRGV. For more information, please contact justin.white@utrgv.edu, william.flores01@utrgv.edu. 


\title{
Unique, Additive, and Interactive Effects of Types of Intimate Partner Cybervictimization on Depression in Hispanic Emerging Adults
}

\author{
Jorge I. Cantu, B.S. \\ Ruby Charak, Ph.D. \\ Department of Psychological Sciences, The University of Texas Rio Grande Valley, Edinburg, \\ Texas
}

\begin{abstract}
APA Citation.
Cantu, J. I. \& Charak R. (2020). Unique, additive, and interactive effects of types of intimate partner cybervictimization on depression in Hispanic emerging adults. Journal of Interpersonal Violence. doi.10.1177/0886260520915552
\end{abstract}

Corresponding author: Ruby Charak, PhD, Assistant Professor, Department of Psychological Science, EIEAB 3.207, The University of Texas Rio Grande Valley. Ph: +1-9566653733. Email: charakruby@gmail.com 


\begin{abstract}
The advent of modern technology has inadvertently created newer avenues for intimate partner victimization to transpire. The present study investigated (i) whether psychological, sexual, and stalking intimate partner cybervictimization (cyber IPV) types were uniquely associated with depression (ii), and whether there were additive, and (iii) interactive effects of cyber IPV types on depression, after controlling for face-to-face IPV among Hispanic emerging adults. Participants were 903 Hispanic emerging adults in the age range of $18-29$ years $(M=20.68, S D$ $=2.42 ; 74 \%$ female) attending a public university in South Texas. Participants completed an online questionnaire assessing cyber IPV types (psychological, sexual, and stalking), face-to-face IPV types (physical, psychological, and sexual) and depression. Results indicated that nearly three-fourths $(73 \%)$ of the sample endorsed at least one type of cyber IPV. Hierarchical regression analysis showed that psychological and sexual cyber IPV was uniquely associated with depression. An accumulating effect (additive effect model) of the three types of cyber IPV on depression was also found. Interaction effect model analysis yielded no significant effects. Findings reveal the detrimental effect of cyber IPV on mental health and calls for institutions and clinicians to develop preventative and treatment programs to aid victim-survivors.
\end{abstract}

Keywords: cyber, intimate partner victimization, depression, Hispanic, emerging adult, additive and interaction effect 


\section{Unique, Additive, and Interactive Effects of Types of Intimate Partner Cybervictimization on Depression in Hispanic Emerging Adults}

Today an increasingly significant aspect of communication in young adults' interpersonal relationships is the use of information and communication technologies (ICTs), including smartphones, the internet, text/instant messaging applications (e.g., WhatsApp), electronic mail, or social media (e.g., Facebook, Twitter, Instagram, Snapchat, blogs). In 2018 it was documented that in the United States, $92 \%$ of millennials, that is, individuals between 23-38 years of age owned a smartphone, $85 \%$ were on social network platforms, and 97\% used the internet (Jiang, 2018). Consequently, the advent of ICTs has inadvertently created newer avenues for interpersonal violence to transpire, including intimate partner violence (e.g., cyber psychological aggression). Presently, the extant literature focuses on face-to-face intimate partner violence and its negative effects on a victim's mental health; in contrast, lesser is known about its cyber variant: intimate partner cyberviolence (C. Brown \& Hegarty, 2018; Gámez-Guadix, Borrajo, \& Calvete, 2018). Despite the limited research, in the past few years there has been noticeable progress in the investigation of cybervictimization by an intimate partner (henceforth referred to as cyber IPV) and the ensuing mental health problems, such as depressive symptoms (Charak, Villarreal, Schmitz, Hirai, \& Ford, 2019; Drouin, Ross, \& Tobin, 2015; Weingarten, Wu, Gates, Carreno, \& Baker, 2018; Wolford-Clevenger et al., 2016).

Cyber IPV is a multidimensional form of violence, akin to face-to-face intimate partner victimization (i.e., face-to-face IPV), comprising psychological, sexual, and stalking victimization (Watkins, Maldonado, \& DiLillo, 2018). Prior studies have demonstrated that the different types of cyber IPV often co-occur (Reed, Tolman, \& Ward, 2017; Watkins, Maldonado, \& DiLillo, 2018; Zweig, Dank, Yahner, \& Lachman, 2013). However, few studies examining the association between cyber IPV types and mental health have investigated the independent effect 
of cyber IPV types. No study to date has examined the unique, additive, nor interactive effect of the three aforementioned types of cyber IPV on mental health problems, such as depression. Notably, most of the studies on cyber IPV focus (except Borrajo, Gámez-Guadix, \& Calvete, 2015) on predominantly White Non-Hispanic samples (e.g., Marganski \& Melander, 2018; Reed et al., 2016); hence, less is known about this form of intimate partner victimization within the Hispanic population.

In 2017, Hispanics were the largest minority group in the U.S. accounting for approximately $18 \%$ of the U.S. population (Noe-Bustamante \& Flores, 2019). Studies on the prevalence of ICTs use among Hispanics indicates that $90 \%$ under the age of 50 years have access to the internet, $80 \%$ use their mobile devices to access the internet, and $65 \%$ of Hispanics are active on social media (A. Brown, López, \& Lopez, 2016; Perrin, 2015). With the prolific usage of ICTs the risk of cyber IPV increases among Hispanic individual as well, particularly during emerging adulthood. This developmental stage is characterized by individuals in the age range of 18-29 years, and is marked by an ambivalence about identifying as an adult, lack of fulfillment of being an independent individual, identity exploration, engaging in risky behavior, and establishment of long-term intimate relationships (Arnett, 2000; Nelson \& Barry, 2005). Its feature of establishing long-term intimate relationships makes it a pivotal period for examining victimization and violence (Borrajo et al., 2015). Moreover, there is evidence that Hispanics experience intimate partner violence and depressive symptoms disproportionately. For example, Caetano and colleagues (2005) found that Hispanics experienced face-to-face IPV twice as more than White Non-Hispanics, after controlling for social economic status. A meta-analysis evaluating the disparity in depressive symptoms between White Non-Hispanic and Hispanics found significantly higher levels of depressive symptoms in Hispanics compared to White Non- 
Hispanics; however, the magnitude of the difference was small (Menselson, Rehkopf, \& Kubzansky, 2008). Thus, the aim of the present study was to add to the growing body of literature on cyber IPV by examining the unique, additive, and interactive effects of three types of cyber IPV, namely, psychological violence, sexual violence, and stalking on depression among Hispanic emerging adults after accounting for the effects of face-to-face physical, psychological, and sexual intimate partner victimization (i.e., face-to-face IPV types).

\section{Cyber IPV}

As mentioned previously, cyber IPV includes, psychological, sexual, and stalking victimization. Psychological cyber IPV is defined by being put down, harassed, insulted, or emotionally hurt by an intimate partner publicizing private or personal information about oneself on social media or group instant/text messaging (e.g., WhatsApp) ICTs, or by information received from an intimate partner through ICTs (Leisring \& Giumetti, 2014; Watkins et al., 2018). Sexual cyber IPV entails being requested or pressurized to send sexual content of oneself or pressured to engage in sexual acts against one's wishes by an intimate partner via ICTs. Additionally, it includes receiving unsolicited sexual content via ICTs from an intimate partner (Watkins et al., 2018; Zweig et al., 2013). Lastly, stalking cyber IPV includes having one's ICTs and accounts accessed by an intimate partner without permission or being monitored by an intimate partner through ICTs and devices, such as a global positioning system (GPS) (Schnurr, Mahatmya, \& Basche, 2013; Watkins et al., 2018).

Literature on cyber IPV conveys that these form of cyber IPV are pervasive among young adults. As an illustration, Zapor and colleagues (2017) conducted a study on college students to examine gender difference in prevalence of lifetime psychological cyber victimization in current dating relationships and found that $74 \%$ of male participants and $72 \%$ of female participants 
reported being the victim of at least one act of technology-mediated dating violence. Within a sample of 342 undergraduate students, it was reported that 1 out of 5 students were victims of online harassment perpetrated by a dating partner (Lindsay, Booth, Messing, \& Thaller, 2016). Another study investigating sexting, including coercing a partner to send sexually explicit photos of themselves, found that $20 \%$ of the sample reported being coerced into it (Drouin et al., 2015). In addition, Reed and colleagues (2015) found that among university students in a dating relationship, $30 \%$ endorsed being victims of electronic intrusion in the past year.

Studies on cyber IPV have conveyed that it is a complex form of intimate partner violence with varied terms, definitions, behaviors, and constructs (C. Brown \& Hegarty, 2018; Gámez-Guadix et al., 2018). An aspect of this complexity can be observed in the varied ways in which cyber IPV instruments have termed and conceptualized this form of violence (C. Brown \& Hegarty, 2018; Gámez-Guadix et al., 2018). For example, Borrajo and colleagues’ (2015) Cyber Dating Abuse Questionnaire gauges cyber dating abuse as a two dimensional form of violence, namely, direct aggression and monitoring/control. Direct aggression comprises ten behaviors that pertain to psychological violence and one that pertain to sexual violence, while monitoring/control was made up of four behaviors that pertained to control and five that pertained to stalking. Related, in the Partner Cyber Abuse Questionnaire, partner cyber abuse is assessed as a unidimensional form of violence (Wolford-Clevenger et al., 2016). The Partner Cyber Abuse Questionnaire comprises nine behaviors that pertain to psychological violence and stalking (Wolford-Clevenger et al., 2016). Using the 34-item Cyber Aggression in Relationship Scale (CARS; Watkins et al., 2018), the present study examined cyber IPV as a multidimensional form of intimate partner violence, comprising psychological violence, sexual 
violence, and stalking as previous research has provided evidence for each as an independent forms of violence (Brem et al., 2017; Reed et al., 2017; Watkins et al., 2018; Zapor et al., 2017).

\section{Co-occurrence between Cyber IPV and Face-to-face IPV}

Cyber IPV possesses key features that differentiate it from face-to-face IPV. First, ICTs facilitates cyber IPV because there is a lack of physical and social cues that tend to occur in faceto-face interaction (Dehue, Bolman, \& Völlink, 2008; Denegri-Knott \& Taylor, 2005). Particularly, individuals may be less inhibited in cyber interactions and enact violent behaviors they would otherwise not commit in face-to-face interactions ( $\mathrm{Li}, 2006)$. Second, ICTs are portable, which allows victims of cyber IPV to experience violent behaviors anywhere, regardless of physical proximity (Runions, Shapka, Dooley, \& Modecki, 2013). The aforementioned features of cyber IPV suggest that its domain and presentation are distinct to those of face-to-face IPV. Acknowledging the differences between these two forms of intimate partner victimization, studies have indicated that victims of cyber IPV were likely to be victims

of face-to-face intimate partner violence (Borrajo, Gámez-Guadix, \& Calvete, 2015; Friz, ClarkCrumpton, Daskaluk, \& Wilson, 2018; Marganski \& Melander, 2018; Reed, Tolman, \& Ward, 2016; Villora, Yubero, \& Navarro, 2019; Zweig et al., 2013). For example, Reed and colleagues (2016) conducted a study investigating the relation between psychological, sexual, and control digital dating abuse (DDA) behaviors and face-to-face psychological, sexual, and stalking IPV and found that DDA victimization were positively associated with face-to-face IPV. These findings on the co-occurrence of cyber IPV and face-to-face IPV raise questions regarding the unique effect of cyber IPV types on one's wellbeing above and beyond the effects of face-to-face IPV types.

\section{Cyber IPV and Mental Health Problems}


Although few in number, studies suggest that cyber IPV is a form of intimate partner victimization that is associated with symptoms of depression and anxiety, symptoms of posttraumatic stress disorder (PTSD), antisocial behavior, and alcohol and substance use (Bennett, Guran, Ramos, \& Margolin, 2011; Borrajo \& Gámez-Guadix, 2016; Dardis, Strauss, \& Gidycz, 2019; Lindsay et al., 2016; Watkins et al., 2018; Weingarten et al., 2018). Drouin, Ross, and Tobin (2015) investigated cyber sexual coercion victimization among young adults and found that it was associated with an increase in symptoms of anxiety, depression, and traumatic stress. Moreover, Sargent and colleagues (2016) conducted a study that examined the relation between cybervictimization, depressive symptoms, and antisocial behavior among first-year college students in a romantic relationship and found that cybervictimization was associated with both depressive symptoms and antisocial behavior. In summary, recent studies on the association between cyber IPV and mental health problems suggest that it is a form of intimate partner violence that warrants further research to comprehend its manifestations and psychological effects, in order to develop efficacious treatment and preventative strategies for victim-survivors. Some studies have examined the association between cyber IPV and depression to accomplish this endeavor; however, no study has investigated the unique, additive, and interactive effects of cyber IPV types on depression after accounting for face-to-face victimization types among Hispanic individuals.

\section{Unique, Additive, and Interactive Effect of Cyber IPV Types}

Past studies have investigated the independent effects of cyber IPV types on mental health problems (Drouin et al., 2015); however, caution must be exercised when interpreting these results, since these studies do not take into account the effects of other potential types of cyber IPV and literature on cyber IPV has found that forms of cyber IPV tend to co-occur (Reed 
et al., 2017). Studies that solely investigate the independent effects of cyber IPV types risk overestimating the effect of a particular cyber IPV type. Therefore, investigating the unique effect, as the present study does, will provide insight into the effect of a cyber IPV type above and beyond the effect of other forms of cyber IPV.

Given that individuals are often exposed to multiple types of cyber IPV, literature on cyber IPV further suffers from an absence of studies focusing on the cumulative and interactive effects of cyber IPV types. Conventionally, studies have examined the effect of multiple types of victimization within interpersonal relationships (e.g., child abuse and face-to-face IPV) on health outcomes via two models, namely, the additive effect model and the interactive effect model (Fujiwara, Okuyama, Izumi, \& Osada, 2010; Senn \& Carey, 2010; Stoicescu, Ameilia, Irwanto, Praptoraharjo, \& Mahanani, 2019). Particularly, the additive effect model is employed to assess the cumulative effect that different forms of victimization may have on an individual's health. While no study to date have utilized the additive effect model to examine cyber IPV types and their association with mental health problems, literature focusing on face-to-face IPV suggests that exposure to multiple types of victimization is associated with an increase in mental health problems, such as depression. For example, in a study examining the additive effects of physical, psychological, and sexual IPV on symptoms of depression and PTSD in the past year, Sabina and Straus (2008) found that exposure to multiple forms intimate partner violence was a predictor of depressive symptoms in women only and a predictor of PTSD symptoms in men and women. The unique feature of the additive effect model is that it weighs all victimization types equally in effecting psychological outcomes.

The interactive effect model overcomes the assumption of equivalency of victimization types and examines interactions between various forms of victimization (e.g., interaction 
between sexual and psychological IPV) and their effect on mental health. Specifically, the interactive effect model suggests that the psychological impact of a specific type of victimization would depend on the level or severity of another type of victimization. To the best of our knowledge, only one study to date has examined the interactive effect of forms of cyber IPV on mental health problems; notably, this study is only available in Spanish language (Borrajo \& Gámez-Guadix, 2016). This study investigated the interactive effect of control and direct aggression (wherein direct aggression comprised one item on sexual aggression and nine items on psychological aggression) cyber dating abuse victimization on depression and anxiety among young adults from Spain and found that control moderated the effect of direct aggression. That is, the association between direct aggression and depression, and anxiety, were much stronger when control was low. However, this study did not account for the confounding effect that faceto-face IPV may have nor did it examine the potential effects of sexual cyber IPV on mental health outcomes, as done in the present study. Examination of the additive and interactive effects of cyber IPV types attempts to identify if there are cumulative or interactive combinations of cyber IPV types that increases the risk of depressive symptoms. Findings will allow clinicians and institutions of higher education to understand specific manifestations and effects of cyber IPV exposure in order to facilitate the development of clinical intervention and prevention programs.

\section{The Current Study}

The present study aimed to examine how cyber IPV types affect symptoms of depression in Hispanic emerging adults.

Aim 1. To examine whether each type of cyber IPV had a significant unique effect on symptoms of depression, after accounting for face-to-face IPV victimization, namely, 
psychological, sexual, and physical violence. Prior studies have established an association between psychological (Wolford-Clevenger et al., 2016), sexual (Drouin et al., 2015), and stalking (Dardis et al., 2019) cyber IPV and depression, although the effect of face-to-face was not taken into account. The present study hypothesized (H1) that psychological, sexual, and stalking cyber IPV each would have a unique effect on depression, after controlling for the effect of face-to-face IPV types, such that those with exposure to any form of cyber IPV would have elevated scores on depression.

Aim 2. To examine whether exposure to more types of cyber IPV would have an additive effect on symptoms of depression, after accounting for face-to-face IPV. To date there is no study that has investigated the additive or cumulative effect of cyber IPV types on depression. Alternatively, studies on face-to-face IPV have indicated that exposure to multiple types of intimate partner victimization increases the risk of depression (Sabina \& Straus, 2008). Contingent on these findings, it was hypothesized (H2) that cyber IPV types would have an accumulating effect on depression in that with every exposure to a different type of cyber IPV there would be an increase in the depression score.

Aim 3. To examine whether the three different types of cyber IPV interact (two- and three-way interactions) to affect depression after accounting for types of face-to-face IPV. To date only one study has provided evidence for a two-way interaction effect of cyber IPV facets on depression and anxiety (Borrajo \& Gámez-Guadix, 2016). Additionally, there are multiple studies on the interaction effects that other types of interpersonal violence victimization (e.g., child abuse and face-to-face IPV) may have on a victim's health and wellbeing (Fujiwara et al., 2010; Kaslow \& Thompson, 2008; Senn \& Carey, 2010; Stoicescu et al., 2019). These studies provide evidence that distinct forms of interpersonal victimization interact to have a differential 
effect on mental health problems. Given that there is only one prior study that examined the interaction effect of two types of cyber IPV, the present study explored two and three-way interaction effects of the three-cyber IPV types on symptoms of depression.

\section{Method}

\section{Participants}

Participants were 912 college-going Hispanic emerging adults enrolled in psychology courses at a University in South Texas situated in a region that is predominantly Hispanic. The inclusionary criteria for the present study were (i) must self-identify as being of Hispanic ethnicity, (ii) an age within range of 18-29 years, and (iii) in a current intimate partner relationship or have been in the past. Participants that did not identify as either male or female were deleted $(N=2)$. After plotting the standardized residuals and predicted values as part of a multiple regression assumption test for the predictor variables, seven cases were identified as outliers (> 3.0 standardized residual). These seven cases were deleted for the final analyses.

The final effective sample consisted of 903 Hispanic emerging adults (74\% female) within an age range of $18-29$ years $(M=20.68, S D=2.42)$ who were at the time of the study in an intimate partner relationship (63.8\%) or have been in the past. Participant's self-reported race were $98 \%$ White, and less than $1 \%$ identified as African-American/Black, American Indian/Alaskan Native, and Native Hawaiian/Other Pacific Islanders, Other, each. Nearly, 91\% of the participants were U.S. citizens by birth; $4.1 \%$ were U.S. citizens by naturalization, and $5.3 \%$ were non-U.S. citizens. The household annual income of $47.4 \%$ of the participants was US\$29,999 and under, $10.9 \%$ had an income between US\$30,000-\$39,000, 10.6\% reported an income in the range of US $\$ 40,000-U S \$ 49,000,9.3 \%$ reported an income between US $\$ 50,000$ - 
$\$ 59,000,4.9 \%$ had an income between US\$60,000-\$69,000, and $16.9 \%$ had an income of US\$70,000 or more.

\section{Procedure}

Participants were recruited through the SONA system, an automated participatory pool management software that assists researchers' setup of studies, recruit participants, and manage course credits or paid participation. On average it took students 45 minutes to complete the survey. Participants who completed the online survey were granted course credit if enrolled in the General Psychology course, and students enrolled in other Psychology courses were granted extra-credit at the discretion of their instructor. To avoid coercion, alternative research activities were available for students who did not want to take the surveys. Data collection for the present study was conducted between Spring 2016 and Spring 2019. The Institutional Review Board at the corresponding author's (RC) university approved the research study.

\section{Measures}

Demographics. Participants reported their gender $(0=$ Male, $1=$ Female $)$, age, relationship status (not currently in an intimate relationship $=0$, currently in an intimate relationship =1), race, citizenship/immigration status, and household income.

Face-to-face intimate partner victimization. To evaluate face-to-face IPV the Conflict Tactic Scale 2 short form (CTS2S) (Straus \& Douglas, 2004) was used. The CTS2S is a five subscale instrument that measures physical assault, psychological aggression, sexual coercion, injury, and negotiation tactics that are used when conflict in intimate relationships arise. The CTS2S measures the perpetration and victimization of these tactics in the past year, or prior to the past year. The physical assault, psychological aggression, and sexual coercion subscales were used for the present study to assess lifetime face-to-face IPV. Victimization for physical assault 
was assessed using two items (e.g., my partner punched or kicked or beat-me-up), two items assessed psychological aggression victimization (e.g., my partner insulted or swore or shouted or yelled at me), and sexual coercion victimization was also assessed via two items (e.g., my partner used force like hitting, holding down, or using a weapon to make me have sex).

Frequency of abuse for victims was rated on an 8-point Likert scale, ranging from 1 (once in the past year) to 8 (this has never happened before); option 7 indicated not in the past year but it did happen before. A dichotomous variable was created for each type of face-to-face IPV such that those who endorsed 1-7 were coded as 1 , that is, there was a presence of a specific type of lifetime IPV, and those who endorsed 8 (i.e., absence of specific lifetime IPV) were coded as 0. Attaining the sample's CTS2S subscales internal consistency reliability coefficient is inappropriate since each subscale is composed of solely two items; however, the instrument has adequate construct and concurrent validity (Straus \& Douglas, 2004).

Cyber intimate partner victimization. The Cyber Aggression in Relationship Scale (CARS) (Watkins et al., 2018) was used to evaluate cyber aggression in intimate relationships. The CARS is an instrument with three subscales that measure psychological, sexual, and stalking cyber IPV in the past year, or prior to the past year. In the present study, the psychological, sexual, and stalking aggression subscales were used to assess lifetime cyber IPV. Psychological cyber IPV was assessed using five items (e.g., my partner sent threatening or harassing messages to me via text or social media), sexual cyber IPV was assessed using four items (e.g., my partner pressured me to send sexual or naked photos of myself to him or her), and lastly, victimization for cyberstalking was assessed via eight items (e. g., my partner checked my phone to see who I was talking to or texting without my permission). Frequency of victimization was rated on a 8-point Likert scale with options ranging from 0 (this has never happened) to 6 (more 
than 20 time in the past year) referring to victimization experienced in the past year, and response 7 (not in the past year, but it did happen before) referring to victimization that happened prior to the past year. A dichotomous variable was created for each type of cyber IPV such that those who endorsed 1-7 were coded as 1 , that is, there was a presence of a specific type of lifetime cyber IPV, and those who endorsed 8 (i.e., absence of specific type of lifetime cyber IPV) were coded as 0 . To analyze the additive effects of lifetime cyber IPV types a composite variable (i.e., cumulative cyber IPV) was created by summing the three different types of cyber IPV experienced. Cumulative cyber IPV ranged from 0 (no cyber IPV) to 3 (three types of cyber $I P V)$. In the development of the CARS, Watkins and colleagues (2018) utilized an item factor analysis and confirmed the presence of three factors, namely, psychological, sexual, and stalking. Moreover, the CARS provide evidence of adequate convergent and discriminant validity for each subscale. Internal consistency reliability for the three subscales in the present sample were $\alpha=$ .81 for psychological cyber IPV, .84 for sexual cyber IPV, and .85 for stalking cyber IPV.

Symptoms of depression. The Patient Health Questionnaire (PHQ) (Kroenke \& Spitzer, 2002) is a nine-item instrument that screens for depression as characterized by the DSM-5 criteria. Frequency of depressive symptoms (e. g., little interest or pleasure in doing things) experienced in the past month was rated on a 4-point Likert scale $(0=$ not at all; $3=$ nearly every day). Responses were summed to provide a PHQ-9 total score. PHQ-9 scores ranged from 0 to 27. Consistent with prior research, a cut-off score of 10 was used to indicate depression (Kroenke, Spitzer, \& Williams, 2001). Increase in PHQ-9 depression severity scores is associated with deteriorating functionality in primary care patients, hence, demonstrating adequate construct validity. Internal consistency reliability for the PHQ-9 scale in the present sample was $\alpha=.89$. 


\section{Data Analysis}

Data were analyzed using SPSS version 25. First, preliminary regression assumption test analyses were conducted to test for violations in linearity, multicollinearity, homoscedasticity, and to identify outliers. Second, descriptive statistics were conducted to examine the distribution of demographic variables, such as gender, relationship status, and face-to-face IPV types, cyber IPV types, and depression within the sample. Second, bivariate correlations were conducted between the demographic variables, face-to-face IPV types, cyber IPV types, and depression. Demographic variables that were significantly associated with the dependent variable (i.e., depression) were used as covariates in subsequent analyses. Third, two separate hierarchical regressions were conducted to examine the variance in depression scores due to (i) the unique effect of each of the three types of cyber IPV; (ii) the two- and three-way interaction effects of cyber IPV types; and (iii) the additive effect of cyber IPV types, after controlling for the effects of covariates, and physical, psychological, and sexual face-to-face IPV types.

Unique and interactive effect of each cyber IPV type. For the regression model analyzing the unique and interactive effect of cyber IPV types on depression, gender and relationship status were entered in Step 1. In Step 2 the types of face-to-face IPV were entered. For examination of the unique effects of the three types of cyber IPV, in Step 3 the three types of cyber IPV were entered. In Step 4, the two-way interactions between the cyber IPV types were entered followed by the three-way interaction between the cyber IPV types in Step 5.

Additive effect of cyber IPV types. To investigate the additive effect of cyber IPV types on depression, first, gender and relationship status were entered in Step 1 of the hierarchical regression analysis. Second the three types of face-to-face IPV were entered in Step 2, and finally, in Step 3 the cumulative cyber IPV variable was entered. 


\section{Results}

\section{Intimate partner victimization types and depression}

Descriptive analysis demonstrated that $24.7 \%$ to $63.3 \%$ of participants endorsed a cyber IPV type. Approximately two-fifths (17.6\%) to a third (32.7\%) of the sample reported experiencing one to three types of cyber IPV. Nearly $21 \%$ to $45 \%$ of participants reported experiencing two specific combinations of cyber IPV types. Additionally, $19.5 \%$ to $57.6 \%$ of the sample endorsed a type of face-to-face IPV. Approximately a third of the sample $(30.5 \%)$ received a PHQ-9 score of 10 and above, indicative of probable depression. The mean score on depression was $7.97(S D=6.08)$. Table 1 gives a detailed account of the distribution for the aforementioned demographic covariates, cyber and face-to-face IPV types, and elevated scores on depression (i.e., cut off score > 9 on PHQ).

\section{Insert Table 1 Here}

\section{Face-to-face and cyber IPV correlations and covariates}

As presented in Table 2, bivariate correlations indicated a significant association between gender, relationship status, and depression, such that female participants and those who were currently not in a relationship were higher on depression scores Additionally, cyber IPV types were positively associated with face-to-face IPV types $\left(r_{\text {range }}=.117\right.$ to .398$)$ indicating that increase in face-to-face cyber IPV types was associated with an increase in types of cyber IPV.

\section{Regression analyses}


In the present sample of Hispanic individuals, those with exposure of either psychological or sexual cyber IPV were at an increased risk of depression, across all levels of the other two types of cyber IPV (step 4; Table 3). This increased risk in depression was present even after accounting for the effects their histories of face-to-face IPV and other forms of cyber IPV on symptoms of depression. Notably, when psychological and sexual cyber IPV are absent, the simple effect of stalking cyber IPV did significantly impact symptoms of depression (step 5; Table 3). The additive hierarchical regression model demonstrated a significant cumulative effect of cyber IPV on depression, after controlling for gender, relationship status, and face-to-face IPV types (Table 4), such that with every exposure to a type of cyber IPV there was 2.15-unit increase in depression score.

\section{Insert Tables 3 and 4 Here}

\section{Discussion}

Studies on cyber IPV among emerging adults indicate that it is a pervasive form of violence that has a detrimental effect on a victim's mental health (Dardis et al., 2019; Drouin et al., 2015; Sargent et al., 2016; Wolford-Clevenger et al., 2016). However, no study to date has investigated the rates of cyber IPV and its effects on depression among Hispanic emerging adults. The present study aimed to extend the literature on cyber IPV by examining the unique, additive, and interactive effect of three types of cyber IPV on depression among Hispanic emerging adults. The take home message from the unique, interactive, and additive effects of cyber IPV types on depression is that psychological and sexual cyber IPV, each had the propensity to increase symptoms of depression (step 4; Table 3), but stalking cyber IPV impacted depression scores only in the absence of both psychological and sexual cyber IPV types 
(step 5; Table 3). Furthermore, findings suggest that the impact of one type of cyber IPV (e.g., sexual cyber IPV) on depression did not depend on the presence of another type of cyber IPV (e.g., psychological cyber IPV) although there was an accumulating effect of the three types of cyber IPV on depression scores. Study findings are discussed below in details.

Although not a hypothesis, the present study found rates of cyber IPV types in Hispanic emerging adults to be in the range among those reported in prior studies. For example, in the present study $53 \%$ of Hispanic emerging adults reported psychological cyber IPV compared to studies reporting 14\% to 77\% among young adults (Bennett et al., 2011; Borrajo, GámezGuadix, \& Calvete, 2015; Lindsay et al., 2016; Watkins et al., 2018; Zapor et al., 2017). Prior studies on young adults reported rates of stalking cyber IPV ranging from $18 \%$ to $75 \%$ (Bennett et al., 2011; Borrajo, Gámez-Guadix, Pereda, et al., 2015; Burke, Wallen, Vail-Smith, \& Knox, 2011; Dardis et al., 2019; Watkins et al., 2018), compared to $63.4 \%$ in the present study. Lastly, rates of sexual cyber IPV across prior studies ranged from $11 \%$ and $32 \%$ among adolescents (Reed et al., 2017; Zweig et al., 2013) and young adults (Drouin et al., 2015; Watkins et al., 2018), compared to $24.6 \%$ in the present study. However, it is important to mention that prior studies use different definitions and instruments/measures to gauge cyber IPV, and hence, the above-mentioned comparisons are limited in this regard. A significant finding of the present study was that $50.2 \%$ of the present sample reported exposure to more than one form of cyber IPV suggesting that victimization of multiple types of cyber IPV are prevalent among Hispanic emerging adults. These rates display the pervasiveness of this form of intimate partner violence among Hispanic emerging adults.

Findings from the regression model partially supported hypothesis-1 indicating that psychological and sexual cyber IPV had a unique effect on depression, beyond the variance in 
depression due to gender, relationship status, and physical, psychological, sexual face-to-face IPV types. Findings are consistent with prior studies that indicate an association between these types of cyber IPV and depression (Borrajo \& Gámez-Guadix, 2016; Drouin et al., 2015; Lindsay et al., 2016; Watkins et al., 2018). In the present sample of Hispanic individuals, the main effect of stalking cyber IPV on depression was non-significant although the simple effect of stalking cyber IPV on depression was significant in the absence of psychological and sexual cyber IPV. Findings are in contrast to prior studies (Borrajo \& Gámez-Guadix, 2016; Dardis et al., 2019; Watkins et al., 2018) that suggest a main effect of stalking cyber IPV on depression. However, these prior studies did not control for the effect of face-to-face IPV types when examining the association between stalking cyber IPV and depression, and their conceptualization of stalking cyber IPV differs. Differences in these factors may confound the results in two ways. First, by not controlling for forms of face-to-face IPV, stalking cyber IPV may have been assumed to be the sole variable leading to the variance in symptoms of depression. Second, different operational definitions of stalking cyber IPV would involve an array of stalking behaviors that may vary in the degree of severity, and ultimately its effect on depression.

In addressing the additive effects of cyber IPV types, the accumulation of cyber IPV types were found to have an additive effect on depression beyond the effect of covariates, and physical, psychological, and sexual face-to-face IPV (hypothesis 2 supported). Particularly, with every additional exposure to a type of cyber IPV, the depression scores increased by more than two units. Findings are consistent with prior studies on face-to-face IPV, which suggest that exposure to multiple forms of intimate partner victimization increases the risk of depression (Dutton, Kaltman, Goodman, Weinfurt, \& Vankos, 2005; Sabina \& Straus, 2008). The additive 
effect of cyber IPV types on depression is in line with the cumulative risk hypothesis (Rutter, 1979) conveying that the experience of multiple forms of cyber violence within the context of an intimate partner relationship is related to a greater risk of developing mental health problems. Hence, as participants in the present study were exposed to more types of cyber IPV, their depression incremented. This observation implies that Hispanic individuals are also subject to the cumulative risk theory suggesting that Hispanic emerging adults with exposure to multiple types of cyber IPV may exhibit exacerbated symptoms of depression. To the best of our knowledge, the present study is the first to examine the additive effect of cyber IPV types on depression and warrant replication. Nonetheless, findings suggest that clinicians should identify individuals with exposure to more than one type of cyber IPV, as they are at an increased risk of depression.

For the interactive effect of cyber IPV types, findings indicated that exposure to specific combinations of cyber IPV types (e.g., sexual and psychological cyber IPV) did not affect depression in Hispanic emerging adults. These findings are in contrast to a prior study that found direct aggression and control cyber dating abuse to have an interaction effect on anxiety and depression (Borrajo \& Gámez-Guadix, 2016). Dissimilar results between studies could be explained by differences in conceptualization of cyber IPV and conception of studies. For instance, the present study defines cyber IPV as a three-factor form of violence (i. e., psychological, sexual, and stalking) instead of a two-factor form of abuse (i.e., direct aggression and monitoring; Borrajo \& Gámez-Guadix, 2016). Additionally, in the present study, sexual cyber IPV was analyzed to identify its potential interaction effects on depression when combined with psychological and stalking cyber IPV. Lastly, the effects of face-to-face IPV types where 
included in the present study's models. In contrast, the aforementioned study did not account for these confounding variables.

The present study findings should be interpreted with the following limitations in mind. First, recall and response biases may exist considering the length of time that may have elapsed between the cyber IPV experience and participation in the study. Second, the design of the study is cross-sectional and the causal relation between cyber IPV types and depression is an assumption based on prior literature (Dardis et al., 2019; Lindsay et al., 2016; WolfordClevenger et al., 2016). Third, prior studies suggest that other types of victimization that are associated with depression, such as peer victimization and child maltreatment, may have confounded the results (Ross, Kaminski, \& Herrington, 2019; Tennant, Demaray, Coyle, \& Malecki, 2015). Fourth, the present study focused on Hispanic emerging adults enrolled at a university, and thus the study findings may not be generalizable to other ethnic/racial groups of emerging adults.

The current findings have significant implications for future research endeavors in the field of cyber IPV in Hispanic emerging adults. The unique effect of psychological and sexual cyber IPV, and the additive effect of the three types of cyber IPV types on depression, shows the importance of examining cyber IPV as a multidimensional form of interpersonal violence among Hispanic individuals as well. While the harmful effect of stalking cyber IPV on depression did not arise in the present study, it is nevertheless a wrongful act. Moreover, studies on cyber IPV should further investigate the mechanistic role of universally established protective (e.g., social support, resilience) and risk factors, (e.g., emotional dysregulation, child maltreatment), and factors associated with the Hispanic culture (e.g., machismo, marianismo) in the association between cyber IPV types and other forms of mental health problems among Hispanic 
individuals. Notably, cultural factors, such as machismo (i.e., male stereotype in Latin culture that encompasses bravado, decision maker of the family), marianismo (i.e., woman's role in Latin American culture in which there is an expectation of submissiveness, modesty, and responsibility for the caretaking of children), and level of acculturation have been found to act as risk and protective factors of face-to-face IPV among Hispanic individuals (Cummings, Gonzalez-Guarda, \& Sandoval, 2013). An understanding of these mechanisms would inform prevention and intervention strategies to combat cyber IPV and related consequences among Hispanic emerging adults. The additive and interactive effects face-to-face IPV and cyber IPV types have on mental health and behavioral problems should be examined, as prior studies have demonstrated that these forms of intimate partner victimization highly co-occur (Marganski \& Melander, 2018; Watkins et al., 2018; Zapor et al., 2017). Additionally, as the present study consists of Hispanic emerging adults between 18 and 29 years old, findings indicate that the importance of this formative developmental period should be affirmed and addressed by clinicians in clinical interventions.

In practice, University institutions should be conscientious about the current pervasive transgressions of cyber IPV among emerging adults. Institutions of higher education should utilize their resources to increase awareness on campus by having mandatory preventative strategies, such as education programs teaching online citizenship and provide training to identify and report cyber IPV (White \& Carmody, 2018). An evidence-based violence intervention, such as the Green-Dot by-stander intervention may be adapted for ICTs and implemented at Universities to address cyber IPV along with face-to-face IPV among young adults (Coker et al., 2015). The bystander intervention emphasizes that communities must be 
aware of the behavioral nature and frequency of violence to safely and effectively intervene in order to mitigate the risk of violence (Coker et al., 2015).

In addition, clinicians who work with emerging adults who have been victims of intimate partner violence and/or are exhibiting depressive symptoms should as well investigate the presence of cyber IPV types and exposure to multiple types of cyber IPV. Clinicians should be culturally sensitive and employ evidence-based therapies, such as cognitive-behavioral therapy to help victim-survivors overcome negative cognitions (e.g., self-blame) that accompany victimization that maybe maintained by cultural factors (e.g. marianismo; Cummings et al., 2013) in order to help mitigate symptoms of depression in Hispanic individuals. Continual research on cyber IPV to refine the etiology and effects on mental and behavioral health is required to develop specified forms of treatment and prevention programs to better aid victimsurvivors.

The present findings warrant replication and future research should continue to investigate the mental health problems that may result from cyber IPV types utilizing the interactive effect model considering that differential effect of specific combinations of other interpersonal violence victimization types (i.e., child abuse and face-to-face IPV) has been reported in prior studies (Fujiwara et al., 2010; Kaslow \& Thompson, 2008; Senn \& Carey, 2010; Stoicescu et al., 2019). In addition, future studies should continue to investigate the association between psychological, sexual, and stalking cyber IPV and mental health using standardized questionnaire to facilitate comparison of findings across samples of White NonHispanics and other diverse and at-risk samples (e.g., LGBTQ+ population, other racial minorities; Charak et al., 2019). In particular, future studies should also focus on examining the severity of cyber IPV as prior studies on adulthood victimization suggest that higher intensities 
of victimization (e.g., longer duration, higher frequency) are as sociated with severe psychological problems (Charak, DiLillo, Messman-Moore, \& Gratz, 2018). 


\section{References}

Arnett, J. J. (2000). Emerging adulthood. A theory of development from the late teens through the twenties. The American Psychologist, 55(5), 469-480.

Bennett, D. C., Guran, E. L., Ramos, M. C., \& Margolin, G. (2011). College students' electronic victimization in friendships and dating relationships: Anticipated distress and associations with risky behaviors. Violence and Victims, 26(4), 410-429.

Borrajo, E., \& Gámez-Guadix, M. (2016). Cyber dating abuse: its link to depression, anxiety and dyadic adjustment. Behavioral Psychology-Psicologia Conductual, 24(2), 221-235.

Borrajo, E., Gámez-Guadix, M., \& Calvete, E. (2015). Cyber dating abuse: Prevalence, context, and relationship with offline dating aggression. Psychological Reports, 116(2), 565-585.

Borrajo, E., Gámez-Guadix, M., Pereda, N., \& Calvete, E. (2015). The development and validation of the cyber dating abuse questionnaire among young couples. Computers in Human Behavior, 48, 358-365.

Brem, M. J., Florimbio, A. R., Grigorian, H., Wolford-Clevenger, C., Elmquist, J., Shorey, R. C., ... Stuart, G. L. (2017). Cyber abuse among men arrested for domestic violence: Cyber monitoring moderates the relationship between alcohol problems and intimate partner violence. Psychology of Violence.

Brown, A., López, G., \& Lopez, M. H. (2016). Digital Divide Narrows for Latinos as More Spanish Speakers and Immigrants Go Online. Retrieved from https://www.pewhispanic.org/2016/07/20/digital-divide-narrows-for-latinos-as-morespanish-speakers-and-immigrants-go-online/

Brown, C., \& Hegarty, K. (2018). Digital dating abuse measures: A critical review. Aggression and Violent Behavior, 40, 44-59.

Burke, S. C., Wallen, M., Vail-Smith, K., \& Knox, D. (2011). Using technology to control intimate partners: An exploratory study of college undergraduates. Computers in Human Behavior, 27(3), 1162-1167.

Caetano, R., Field, C. A., Ramisetty-Mikler, S., \& McGrath, C. (2005). The 5-year course of intimate partner violence among White, Black, and Hispanic couples in the United States. Journal of Interpersonal Violence, 20(9), 1039-1057. 
Charak, R., DiLillo, D., Messman-Moore, T. L., \& Gratz, K. L. (2018). Latent classes of lifetime sexual victimization characteristics in women in emerging adulthood: Differential relations with emotion dysregulation. Psychology of Violence, 8(5), 570-579.

Charak, R., Villarreal, L., Schmitz, R. M., Hirai, M., \& Ford, J. D. (2019). Patterns of childhood maltreatment and intimate partner violence, emotion dysregulation, and mental health symptoms among lesbian, gay, and bisexual emerging adults: A three-step latent class approach. Child Abuse \& Neglect, 89, 99-110.

Coker, A. L., Fisher, B. S., Bush, H. M., Swan, S. C., Williams, C. M., Clear, E. R., \& DeGue, S. (2015). Evaluation of the Green Dot Bystander Intervention to reduce interpersonal violence among college students across three campuses. Violence against Women, 21(12), 1507-1527.

Cummings, A. M., Gonzalez-Guarda, R. M., \& Sandoval, M. F. (2013). Intimate partner violence among Hispanics: A review of the literature. Journal of Family Violence, 28(2), 153-171.

Dardis, C. M., Strauss, C. V., \& Gidycz, C. A. (2019). The psychological toll of unwanted pursuit behaviors and intimate partner violence on undergraduate women: A dominance analysis. Psychology of Violence, 9(2), 209-220.

Dehue, F., Bolman, C., \& Völlink, T. (2008). Cyberbullying: Youngsters' experiences and parental perception. CyberPsychology \& Behavior, 11(2), 217-223.

Denegri-Knott, J., \& Taylor, J. (2005). The labeling game: A conceptual exploration of deviance on the internet. Social Science Computer Review, 23(1), 93-107.

Drouin, M., Ross, J., \& Tobin, E. (2015). Sexting: A new, digital vehicle for intimate partner aggression? Computers in Human Behavior, 50, 197-204.

Dutton, M. A., Kaltman, S., Goodman, L. A., Weinfurt, K., \& Vankos, N. (2005). Patterns of intimate partner violence: Correlates and outcomes. Violence and Victims, 20(5), 483497.

Friz, P. A. T., Clark-Crumpton, J., Daskaluk, S., \& Wilson, L. (2018). Electronic partner aggression victimization: Its frequency and relation to offline partner aggression and psychological functioning. Partner Abuse, 9(4), 421-438. 
Fujiwara, T., Okuyama, M., Izumi, M., \& Osada, Y. (2010). The impact of childhood abuse history and domestic violence on the mental health of women in Japan. Child Abuse \& Neglect, 34(4), 267-274.

Gámez-Guadix, M., Borrajo, E., \& Calvete, E. (2018). Partner abuse, control and violence through internet and smartphones: Characteristics, evaluation and prevention. Papeles Del Psicologo, 39(3), 218-227.

Jiang, J. (2018). Millennials stand out for their technology use, but older generations also embrace digital life. Retrieved from https://www.pewresearch.org/fact$\underline{\operatorname{tank} / 2018 / 05 / 02 / \text { millennials-stand-out-for-their-technology-use-but-older-generations- }}$ also-embrace-digital-life/

Kaslow, N. J., \& Thompson, M. R. (2008). Associations of child maltreatment and intimate partner violence with psychological adjustment among low SES, African American children. Child Abuse \& Neglect, 32(9), 888-896.

Kroenke, K., \& Spitzer, R. L. (2002). The PHQ-9: A new depression diagnostic and severity measure. Psychiatric Annals, 32(9), 509-515.

Kroenke, K., Spitzer, R. L., \& Williams, J. B. W. (2001). The PHQ-9. Journal of Internal Medicine, 16(9), 606-613.

Leisring, P. A., \& Giumetti, G. W. (2014). Sticks and stones may break my bones, but abusive text messages also hurt: Development and validation of the Cyber Psychological Abuse scale. Partner Abuse, 5(3), 323-341.

Li, Q. (2006). Cyberbullying in schools: A research of gender differences. School Psychology International, 27(2), 157-170.

Lindsay, M., Booth, J. M., Messing, J. T., \& Thaller, J. (2016). Experiences of online harassment among emerging adults: Emotional reactions and the mediating role of fear. Journal of Interpersonal Violence, 31(19), 3174-3195.

Marganski, A., \& Melander, L. (2018). Intimate partner violence victimization in the cyber and real world: Examining the extent of cyber aggression experiences and its association with in-person dating violence. Journal of Interpersonal Violence, 33(7), 1071-1095.

Menselson, T., Rehkopf, D. H., \& Kubzansky, L. D. (2008). Depression among Latinos in the United States: A meta-analytic review. Journal of Consulting and Clinical Psychology, 76(3), 355-366. 
Noe-Bustamante, L., \& Flores, A. (2019). Facts on Latinos in the U.S. Retrieved from https://www.pewresearch.org/hispanic/fact-sheet/latinos-in-the-u-s-fact-sheet/

Perrin, A. (2015). Social Media Usage: 2005-2015. Retrieved from https://www.pewinternet.org/2015/10/08/social-networking-usage-2005-2015/

Reed, L. A., Tolman, R. M., \& Safyer, P. (2015). Too close for comfort: Attachment insecurity and electronic intrusion in college students' dating relationships. Computers in Human Behavior, 50, 431-438.

Reed, L. A., Tolman, R. M., \& Ward, L. M. (2016). Snooping and sexting: Digital media as a context for dating aggression and abuse among college students. Violence against Women, 22(13), 1556-1576.

Reed, L. A., Tolman, R. M., \& Ward, L. M. (2017). Gender matters: Experiences and consequences of digital dating abuse victimization in adolescent dating relationships. Journal of Adolescence, 59, 79-89.

Ross, N. D., Kaminski, P. L., \& Herrington, R. (2019). From childhood emotional maltreatment to depressive symptoms in adulthood: The roles of self-compassion and shame. Child Abuse \& Neglect, 92, 32-42.

Runions, K., Shapka, J. D., Dooley, J., \& Modecki, K. (2013). Cyber-aggression and victimization and social information processing: Integrating the medium and the message. Psychology of Violence, 3(1), 9-26.

Rutter, M. (1979). Protective factors in children's responses to stress and disadvantage. Annals Of The Academy Of Medicine, Singapore, 8(3), 324-338.

Sabina, C., \& Straus, M. A. (2008). Polyvictimization by dating partners and mental health among US college students. Violence and Victims, 23(6), 667-682.

Sargent, K. S., Krauss, A., Jouriles, E. N., \& McDonald, R. (2016). Cyber victimization, psychological intimate partner violence, and problematic mental health outcomes among first-year college students. Cyberpsychology, Behavior, and Social Networking, 19(9), 545-550.

Schnurr, M. P., Mahatmya, D., \& Basche, R. A., III. (2013). The role of dominance, cyber aggression perpetration, and gender on emerging adults' perpetration of intimate partner violence. Psychology of Violence, 3(1), 70-83. 
Senn, T. E., \& Carey, M. P. (2010). Child maltreatment and women's adult sexual risk behavior: Childhood sexual abuse as a unique risk factor. Child Maltreatment, 15(4), 324-335.

Stoicescu, C., Ameilia, R., Irwanto, Praptoraharjo, I., \& Mahanani, M. (2019). Syndemic and synergistic Eeffects of intimate partner violence, crystal methamphetamine, and depression on HIV sexual risk behaviors among women who inject drugs in Indonesia. Journal Of Urban Health: Bulletin Of The New York Academy Of Medicine.

Straus, M. A., \& Douglas, E. M. (2004). A short form of the Revised Conflict Tactics Scales, and typologies for severity and mutuality. Violence and Victims, 19(5), 507-520.

Tennant, J. E., Demaray, M. K., Coyle, S., \& Malecki, C. K. (2015). The dangers of the web: Cybervictimization, depression, and social support in college students. Computers in Human Behavior, 50, 348-357.

Villora, B., Yubero, S., \& Navarro, R. (2019). Associations between feminine gender norms and cyber dating abuse in female adults. Behavioral Sciences, 9(4).

Watkins, L. E., Maldonado, R. C., \& DiLillo, D. (2018). The Cyber Aggression in Relationships Scale: A new multidimensional measure of technology-based intimate partner aggression. Assessment, 25(5), 608-626.

Weingarten, C., Wu, A., Gates, K., Carreno, P., \& Baker, C. (2018). The association between electronic and in-person dating violence victimization, anxiety, and depression among college students in Hawai'i. Partner Abuse, 9(4), 313-334.

White, W. E., \& Carmody, D. (2018). Preventing online victimization: College students' views on intervention and prevention. Journal of Interpersonal Violence, 33(14), 2291-2307.

Wolford-Clevenger, C., Zapor, H., Brasfield, H., Febres, J., Elmquist, J., Brem, M., . . Stuart, G. L. (2016). An examination of the Partner Cyber Abuse Questionnaire in a college student sample. Psychology of Violence, 6(1), 156-162.

Zapor, H., Wolford-Clevenger, C., Elmquist, J., Febres, J., Shorey, R. C., Brasfield, H., . . . Stuart, G. L. (2017). Psychological aggression committed through technology: A study with dating college students. Partner Abuse, 8(2), 127-145.

Zweig, J. M., Dank, M., Yahner, J., \& Lachman, P. (2013). The rate of cyber dating abuse among teens and how it relates to other forms of teen dating violence. Journal of Youth and Adolescence, 42(7), 1063-1077. 


\section{Table 1.}

Covariates, Cyber IPV, Face-to-Face IPV, and Elevated Depression in Hispanic Emerging Adults.

\begin{tabular}{lcc}
\hline Study variables & $n$ & Percentage \\
\hline Gender & & \\
Male & 234 & 25.9 \\
Female & 669 & 74.1 \\
Relationship status & 576 & 63.8 \\
Currently in an intimate relationship & 327 & 36.2 \\
Not currently in an intimate relationship & & 52.9 \\
Cyber IPV & 478 & 24.7 \\
Psychological cyber IPV & 223 & 63.3 \\
Sexual cyber IPV & 572 & 22.8 \\
Stalking cyber IPV & 206 & 32.7 \\
Single type of cyber IPV & 295 & 17.6 \\
Two types of cyber IPV & 159 & 19.7 \\
Three types of cyber IPV & 178 & 45.1 \\
Psychological + sexual cyber IPV & 407 & 20.7 \\
Psychological + stalking cyber IPV & 187 & \\
Sexual + stalking cyber IPV & & 57.6 \\
Face-to-face IPV & 520 & 19.5 \\
Psychological IPV & 176 & 23.9 \\
Physical IPV & 216 & 30.5 \\
Sexual IPV & 315 & \\
Elevated depression & & \\
\hline Note. Elevated depression indicates participants
\end{tabular}

Note. Elevated depression indicates participants who obtained a score of 10 and above in the PHQ-9. IPV = intimate partner victimization; PHQ = Patient Health Questionnaire. 
Table 2. Correlation Between Study Variables.

\begin{tabular}{|c|c|c|c|c|c|c|c|c|c|}
\hline & 8 \\
\hline 1. & Gender & - & & & & & & & \\
\hline 2. & Relationship status & -.043 & - & & & & & & \\
\hline 3. & $\begin{array}{l}\text { Depression } \\
(6.08)\end{array}$ & $.121 * * *$ & $.117 * *$ & - & & & & & \\
\hline 4. & Psychology cyber IPV & .009 & .041 & $.221 * * *$ & - & & & & \\
\hline 5. & Sexual cyber IPV & .057 & $.188 * * *$ & $.189 * * *$ & $.308 * * *$ & - & & & \\
\hline 6. & Stalking cyber IPV & -.004 & -.044 & $.168 * * *$ & $.480 * * *$ & $.244 * * *$ & - & & \\
\hline 7. & Psychological F2F IPV & -.012 & -.057 & $.135^{* * *}$ & $.398 * * *$ & $.117 * * *$ & $.389 * * *$ & - & \\
\hline 8. & Physical F2F IPV & -.015 & -.004 & $.079 *$ & $.223 * * *$ & $.172 * * *$ & $.223 * * *$ & $.388 * * *$ & - \\
\hline 9. & Sexual F2F IPV & .041 & $.107 * *$ & $.138 * * *$ & $.227 * * *$ & $.317 * * *$ & $.222 * * *$ & $.261 * * *$ & $.373 * * *$ \\
\hline
\end{tabular}

Note. For gender, $0=$ male and $1=$ female. For relationship status, $0=$ currently in an intimate relationship and $1=$ not currently in an intimate relationship. IPV = intimate partner victimization; F2F = face-to-face.

$* p<.05 . * * p<.01 . * * * p<.001$. 
Table 3.

Hierarchical Regression Examining the Unique and Interactive Effects of Cyber IPV Types on Depression After Controlling for the Effects of Covariates and Face-to-Face IPV in Hispanic Emerging Adults.

\begin{tabular}{|c|c|c|c|c|c|c|c|}
\hline Study variables & $R$ & $R^{2}$ & $\Delta R^{2}$ & $F$ & $d f$ & $B$ & $\beta$ \\
\hline Step 1 & .172 & .030 & .030 & $13.765 * * *$ & 2,900 & & \\
\hline Gender & & & & & & 1.755 & $.127 * * *$ \\
\hline Relationship status & & & & & & 1.549 & $.122 * * *$ \\
\hline Step 2 & .240 & .057 & .028 & $10.930 * * *$ & 5,897 & & \\
\hline Gender & & & & & & 1.722 & $.124 * * *$ \\
\hline Relationship status & & & & & & 1.515 & $.120 * * *$ \\
\hline Psychological F2F IPV & & & & & & 1.462 & $.119 * *$ \\
\hline Physical F2F IPV & & & & & & 0.036 & -.002 \\
\hline Sexual F2F IPV & & & & & & 1.256 & $.088 *$ \\
\hline Step 3 & .307 & .094 & .037 & $11.609 * * *$ & 8,12894 & & \\
\hline Gender & & & & & & 1.638 & $.118 * * *$ \\
\hline Relationship status & & & & & & 1.273 & $.101 * *$ \\
\hline Psychological F2F IPV & & & & & & 0.587 & .048 \\
\hline Physical F2F IPV & & & & & & -0.183 & -.012 \\
\hline Sexual F2F IPV & & & & & & 0.597 & .042 \\
\hline Psychological cyber IPV & & & & & & 1.607 & $.132 * *$ \\
\hline Sexual cyber IPV & & & & & & 1.267 & $.090 *$ \\
\hline Stalking cyber IPV & & & & & & 0.793 & .063 \\
\hline Step 4 & .311 & .097 & .003 & $8.693 * * *$ & 11,891 & & \\
\hline Gender & & & & & & 1.613 & $.116^{* * *}$ \\
\hline Relationship status & & & & & & 1.310 & $.104 * *$ \\
\hline Psychological F2F IPV & & & & & & 0.598 & .049 \\
\hline Physical F2F IPV & & & & & & -0.125 & -.008 \\
\hline Sexual F2F IPV & & & & & & 0.567 & .040 \\
\hline Psychological cyber IPV & & & & & & 1.910 & $.157^{*}$ \\
\hline Sexual cyber IPV & & & & & & 2.104 & .149 \\
\hline Stalking cyber IPV & & & & & & 1.342 & $.106^{*}$ \\
\hline $\begin{array}{l}\text { Psychological } \times \text { Sexual cyber } \\
\text { IPV }\end{array}$ & & & & & & 0.649 & .042 \\
\hline $\begin{array}{l}\text { Psychological } \times \text { Stalking cyber } \\
\text { IPV }\end{array}$ & & & & & & -0.698 & -.057 \\
\hline Sexual $\times$ Stalking cyber IPV & & & & & & -1.642 & -.110 \\
\hline Step 5 & .313 & .098 & .001 & $8.052 * * *$ & 12,890 & & \\
\hline Gender & & & & & & 1.614 & $.116^{* * *}$ \\
\hline Relationship status & & & & & & 1.300 & $.103 * *$ \\
\hline Psychological F2F IPV & & & & & & 0.606 & .049 \\
\hline Physical F2F IPV & & & & & & -0.156 & -.010 \\
\hline Sexual F2F IPV & & & & & & 0.602 & .042 \\
\hline Psychological cyber IPV & & & & & & 2.220 & $.182 *$ \\
\hline Sexual cyber IPV & & & & & & 2.948 & $.209 *$ \\
\hline Stalking cyber IPV & & & & & & 1.491 & $.118 *$ \\
\hline $\begin{array}{l}\text { Psychological } \times \text { Sexual cyber } \\
\text { IPV }\end{array}$ & & & & & & -1.171 & -.077 \\
\hline $\begin{array}{l}\text { Psychological } \times \text { Stalking cyber } \\
\text { IPV }\end{array}$ & & & & & & -1.160 & -.095 \\
\hline Sexual $\times$ Stalking cyber IPV & & & & & & -3.067 & -.205 \\
\hline $\begin{array}{l}\text { Psychological } \times \text { Sexual } \times \\
\text { Stalking cyber IPV }\end{array}$ & & & & & & 2.535 & 159 \\
\hline
\end{tabular}


Table 4.

Hierarchical Regression Examining the Association Between Cumulative Cyber IPV and Depression After Controlling for the Effects of Covariates and Face-to-Face Psychological, Physical, and Sexual IPV in Hispanic Emerging Adults.

\begin{tabular}{|c|c|c|c|c|c|c|c|}
\hline Study variables & $R$ & $R^{2}$ & $\Delta R^{2}$ & $F$ & $d f$ & $B$ & $\beta$ \\
\hline Step 1 & .172 & .030 & .030 & $13.765 * * *$ & 2,900 & & \\
\hline Gender & & & & & & 1.755 & $.127 * * *$ \\
\hline Relationship status & & & & & & 1.549 & $.122 * * *$ \\
\hline Step 2 & .240 & .057 & .028 & $10.930 * * *$ & 5,897 & & \\
\hline Gender & & & & & & 1.722 & $.124 * * *$ \\
\hline Relationship status & & & & & & 1.515 & $.120 * * *$ \\
\hline Psychological F2F IPV & & & & & & 1.462 & $.119 * *$ \\
\hline Physical F2F IPV & & & & & & 0.036 & .002 \\
\hline Sexual F2F IPV & & & & & & 1.256 & $.088 *$ \\
\hline Step 3 & .305 & .093 & .036 & $15.308 * * *$ & 6,896 & & \\
\hline Gender & & & & & & 1.648 & $.119 * * *$ \\
\hline Relationship status & & & & & & 1.315 & $.104 * *$ \\
\hline Psychological F2F IPV & & & & & & 0.580 & .047 \\
\hline Physical F2F IPV & & & & & & -0.185 & -.012 \\
\hline Sexual F2F IPV & & & & & & 0.597 & .042 \\
\hline Cumulative cyber IPV & & & & & & 1.230 & $.215 * * *$ \\
\hline
\end{tabular}

Note. IPV = intimate partner victimization; F2F = face-to-face.

$* p<.05 . * * p<.01 . * * * p<.001$. 